\title{
Relapse Rate in Survivors of Acute Autoimmune Thrombotic Thrombocytopenic Purpura Treated with or without Rituximab
}

\author{
Tanja Falter ${ }^{1,2, *}$ Stephanie Herold ${ }^{1, *}$ Veronika Weyer-Elberich ${ }^{3}$ Carina Scheiner ${ }^{3}$ \\ Veronique Schmitt ${ }^{1}$ Charis von Auer ${ }^{4}$ Xavier Messmer ${ }^{2,4}$ Philipp Wild ${ }^{2}$ Karl J. Lackner ${ }^{1}$ \\ Bernhard Lämmle 2,5 Inge Scharrer ${ }^{4}$
}
${ }^{1}$ Institute of Clinical Chemistry and Laboratory Medicine, University Medical Center of the Johannes Gutenberg University, Mainz, Rheinland-Pfalz, Germany
${ }^{2}$ Center for Thrombosis and Hemostasis, University Medical Center of the Johannes Gutenberg University, Mainz, Rheinland-Pfalz, Germany
3 Institute of Medical Biostatistics, Epidemiology and Informatics, University Medical Center of the Johannes Gutenberg University, Mainz, Rheinland-Pfalz, Germany
${ }^{4}$ Department of Hematology, Oncology and Pneumology, University Medical Center of the Johannes Gutenberg University, Mainz, Rheinland-Pfalz, Germany
${ }^{5}$ University Clinic of Hematology \& Central Hematology Laboratory, Bern University Hospital, University of Bern, Bern, Switzerland

\begin{abstract}
Address for correspondence Tanja Falter, PhD, Institute of Clinical Chemistry and Laboratory Medicine, University Medical Center of the Johannes Gutenberg University, Langenbeckstraße 1, 55131 Mainz, Rheinland-Pfalz, Germany

(e-mail: tanja.falter@unimedizin-mainz.de).
\end{abstract}

Thromb Haemost 2018;118:1743-1751.

\begin{abstract}
Keywords

- ADAMS/ADAMTS13

- thrombotic thrombocytopenic purpura (TTP/HUS)

- clinical studies

- autoantibodies

Background Autoimmune thrombotic thrombocytopenic purpura (iTTP) is caused by autoantibody-mediated severe a disintegrin and metalloprotease with thrombospondin type 1 repeats, member 13 (ADAMTS13) deficiency leading to micro-angiopathic haemolytic anaemia (MAHA) and thrombocytopenia with organ damage. Patients survive with plasma exchange (PEX), fresh frozen plasma replacement and corticosteroid treatment. Anti-CD20 monoclonal antibody rituximab is increasingly used in patients resistant to conventional PEX or relapsing after an acute bout.

Objective This retrospective observational study focused on the relapse rate and possible influencing factors including treatment with rituximab first introduced in 2003.

Patients and Methods Seventy patients treated between January 2003 and November 2014 were evaluated. Number, duration, clinical manifestations, laboratory data and treatment of acute episodes were documented. Diagnostic criteria of acute iTTP were thrombocytopenia, MAHA, increased lactate dehydrogenase and severe ADAMTS13 deficiency.

Results Fifty-four female and 16 male patients had a total of 224 acute episodes over a median observation period of 8.3 years. The relapse rate was $2.6 \%$ per month, for women $2.4 \%$ and for men $3.5 \%$ per month. Since 2003, 17 patients with a first iTTP episode were treated with rituximab, whereas 28 were not. There was a trend towards lower relapse rates after rituximab treatment over the ensuing years. However, this was statistically not significant.
\end{abstract}

* Tanja Falter and Stephanie Herold are first co-authors of the study.

received

February 19, 2018

accepted after revision July 11,2018
DOI https://doi.org/ 10.1055/s-0038-1668545. ISSN 0340-6245.
(C) 2018 Georg Thieme Verlag KG Stuttgart · New York
License terms

() (1) $\Theta \circledast$ 
Conclusion This analysis does not show a significant reduction of acute iTTP relapses by rituximab given during an acute bout. Initial episodes are characterized by more severe clinical signs compared with the less severe relapses. Furthermore, men suffer significantly more frequent and considerably more serious acute relapses.

\section{Introduction}

Autoimmune thrombotic thrombocytopenic purpura (iTTP) is an acute, life-threatening disorder, and survivors are at risk of disease relapse. Acute episodes are characterized by consumptive thrombocytopenia, micro-angiopathic haemolytic anaemia (MAHA) and spontaneous von Willebrand factor (VWF)-induced platelet clumping. A severe a disintegrin and metalloprotease with thrombospondin type 1 repeats, member 13 (ADAMTS13) deficiency $(<10 \%)$ due to autoantibodies against ADAMTS13 is associated with acute episodes. ADAMTS13 regulates the size of the newly synthesized and secreted ultra-large (UL) VWF multimers, and cleaves these into less haemostatically active forms. ${ }^{1,2}$ Symptoms of iTTP are highly variable. Many patients suffer from neurological symptoms such as confusion, headache, paresis, aphasia and coma. Thrombocytopenia can result in petechiae, epistaxis and gingival bleeding. Further symptoms are abdominal pain, nausea, fatigue, proteinuria and cardiac complications. ${ }^{3}$ Patients surviving an acute iTTP bout and showing normalization of the laboratory parameters are often considered to be cured; however, recurrences of acute iTTP attacks are common. ${ }^{2,4}$

Since 1991, plasma exchange (PEX) and corticosteroids are the primary standard of care in iTTP. ${ }^{5,6}$ However, there is a proportion of iTTP patients having refractory acute episodes, exacerbations or a high tendency to relapse. Since 2002, rituximab, an anti-CD20 monoclonal antibody, was used in the treatment of iTTP and has become an international standard of therapy. ${ }^{7}$ PEX removes ADAMTS13 autoantibodies as well as UL VWF multimers and fresh frozen plasma (FFP) replacement supplies ADAMTS13. Efficacy of PEX reducing mortality in acute episodes from 90 to 20 to $30 \%$ is indisputable $3,4,6$; nevertheless, PEX and corticosteroids are not as efficient in suppressing autoantibodies as rituximab. Rituximab suppresses the disease-associated ADAMTS13 inhibitor production by depleting B lymphocytes. ${ }^{8}$ Despite widespread application in iTTP, rituximab is still an off-label use.

In the current retrospective observational study, we investigated iTTP patients focusing on the relapse rate and possible influencing factors including treatment with rituximab.

\section{Patients and Methods}

In this systematic retrospective study, we analysed all iTTP patients referred to the University Medical Center (UMC) Mainz from January 2003 to November 2014. Subjects had an acute iTTP bout or were consulting our institution having survived an earlier acute iTTP episode. Inclusion criteria were the clinical diagnosis of iTTP, defined as thrombocytopenia
( $<150,000 / \mu \mathrm{L})$, MAHA, increased lactate dehydrogenase (LDH; > 1.5 $\times$ upper limit of normal values) with or without ischaemic organ damage. Since 2003, severe ADAMTS13 deficiency $(<10 \%)$ caused by an ADAMTS13 autoantibody during an acute bout is an additional diagnostic requirement for iTTP.

Acute iTTP episodes were treated according to a standard procedure of the UMC Mainz and in accordance with the international guidelines. All iTTP patients received PEX using FFP or Octaplas SD (Octapharma, Vienna, Austria) daily from admission until platelet count of $>150,000 / \mu \mathrm{L}$ was reached for longer than 48 hours. From the first day of acute iTTP corticosteroids, usually prednisolone, 1 to $2 \mathrm{mg} / \mathrm{kg}$ body weight, were given daily.

In off-label use, rituximab was administered for the first time in 2003. In this iTTP cohort, rituximab (MabThera; Roche, Grenzach-Wyhlen, Germany) was only used in acute iTTP bouts (1-4 weekly infusions of $375 \mathrm{mg} / \mathrm{m}^{2}$ each). In most cases, rituximab was administered to patients with thrombocytopenia persisting under daily PEX for $\geq 5$ days. A second indication for rituximab treatment was in relapsing iTTP patients without severe organ manifestation where rituximab was given to avoid PEX.

Complete remission was defined as full resolution of the clinical manifestations, especially neurological symptoms, with normalized platelet count for more than 30 consecutive days after the last PEX. ${ }^{9}$

This retrospective study was approved by German law (Landeskrankenhausgesetz §36 and §37) in accordance with the Declaration of Helsinki and by the Ethics Committee of “Landesärztekammer Rheinland-Pfalz" [837.506.15 (10274)].

\section{Assays}

If plasma ADAMTS13 activity was measured between 1996 and 2002, the VWF multimer degradation method (immunoblotting) was used. ${ }^{1,4}$ Since January 2003, ADAMTS13 activity was examined by the residual ristocetin co-factor-based method. ${ }^{10}$ Since April 2010, it was examined by the fluorescence resonance energy transfer system (FRETS-VWF73) method $^{11}$ modified according to Kremer Hovinga et al. ${ }^{12}$ ADAMTS13 activity was expressed as percentage relative to that of pooled normal plasma. The normal range of ADAMTS13 activity in the ristocetin co-factor-based method was defined as 52 to $134 \%$ in 80 healthy controls with a detection limit of $6.25 \%$. The normal range for the FRETS-VWF73 assay in healthy donors is $>50 \%$ with a limit of detection of $1 \%{ }^{4,12}$

ADAMTS13 inhibitor was detected by incubating a mixture of heat-inactivated patient plasma with pooled normal plasma $(1: 1 ; \mathrm{v}: \mathrm{v})$ for 60 minutes at $37^{\circ} \mathrm{C}$ and then measuring the ADAMTS13 activity by the FRETS-VWF73 assay. ${ }^{12}$ Inhibition of $50 \%$ of normal plasma ADAMTS13 activity by undiluted patient 
plasma was defined as 1 Bethesda unit (BU) per mL. An ADAMTS13 inhibitor was diagnosed when $\geq 0.5 \mathrm{BU} / \mathrm{mL}$ were found.

\section{Variables/Covariates}

The following data of iTTP patients were collected: date of birth, sex, number of biological children, body height and weight, calculated body mass index, smoking status and possible co-morbidities.

Data concerning the acute iTTP bouts of each patient was collected such as number of acute bouts, clinical symptoms, beginning and end of the respective bout (admission into and discharge from a hospital), calculated time of an acute episode, calculated age at a bout and calculated whole observation time. Therapeutic procedures (PEX, immune adsorption or splenectomy) and medication (corticosteroids, rituximab, other) given during an acute episode were documented.

Furthermore, we collected laboratory data such as platelet count, LDH, haemoglobin, presence of schistocytes, ADAMTS13 activity and ADAMTS13 inhibitors.

\section{Clinical Severity Score}

To classify the severity of an iTTP bout, we established a score based on clinical and laboratory data ( - Table 1 ). It divides all iTTP bouts into five categories. Category 0 defines acute episodes characterized by all laboratory abnormalities listed in - Table 1 only without manifest clinical signs, usually encountered in known iTTP patients during outpatient visits. Category 1 involves mild clinical symptoms and all the laboratory abnormalities as in category 0. Category 2 and category 3 include more severe clinical signs and symptoms. Acute iTTP episodes with fatal outcome were grouped in category 4 (-Table 1 ).

\section{Statistical Analysis}

We organized all data in a SPSS file pseudonymizing patient names according to the guidelines of the ethics committee.

For descriptive analysis, median and interquartile range (IQR), as well as minimum and maximum were calculated for continuous variables. In addition, absolute and relative frequencies were computed for categorical variables and visualized via bar charts. For interpreting the recurrent events, a graphic is created with one line for each patient on the $y$-axis with dots for each recurring event with observation time on the $x$-axis. Kaplan-Meier estimates were used to describe the relapse-free survival times for patients who did or did not receive rituximab. The log-rank test was used to compare the curves. Additionally, a Cox proportional hazard regression model was used to evaluate the effect of explorative variables on relapse-free survival.

To model the number of events for each patient and specifically to estimate the relapse rate in different subgroups, a Poisson model with the log-transformed observation time incorporated as offset variable was performed.

For confirmatory analysis to estimate the rate ratio for patients treated with or without rituximab, adjusting for the variables sex and age, an Anderson-Gill model for the recurrent events was performed. The Poisson model and the AndersonGill model take into account the dependency between all iTTP
Table 1 Clinical severity score

\begin{tabular}{|c|c|}
\hline $\begin{array}{l}0=\text { laboratory } \\
\text { abnormalities } \\
\text { only }\end{array}$ & $\begin{array}{l}\text { All four laboratory abnormalities listed } \\
\text { must be present } \\
\text { - thrombocytopenia }(<150,000 / \mu \mathrm{L}) \\
\text { - increased LDH ( }>1.5 \times \text { upper limit } \\
\text { of normal) } \\
\text { - decreased haemoglobin }(<12 \mathrm{~g} / \mathrm{dL} \\
\text { in females, }<14 \mathrm{~g} / \mathrm{dL} \text { in males) } \\
\text { - presence of schistocytes }\end{array}$ \\
\hline $1=$ mild & $\begin{array}{l}\text { Laboratory abnormalities plus at least } \\
\text { one of the following clinical } \\
\text { manifestations } \\
\text { - haematoma, petechiae, ecchymoses } \\
\text { - cephalgia, vertigo, nausea } \\
\text { - fatigue, drowsiness, weakness } \\
\text { - (sub-)febrile temperatures, shivering } \\
\text { - pain, especially abdominal pain }\end{array}$ \\
\hline $2=$ moderate & $\begin{array}{l}\text { Laboratory abnormalities plus at least } \\
\text { one of the following clinical } \\
\text { manifestations } \\
\text { - micro- or macro-haematuria } \\
\text { - icterus } \\
\text { - tachycardia, dyspnoea, } \\
\text { - reversible dys- or paresthesia, } \\
\text { visual field defects } \\
\text { - impaired consciousness } \\
\text { (somnolence, stupor), disorientation }\end{array}$ \\
\hline $3=$ severe & $\begin{array}{l}\text { Laboratory abnormalities plus at least } \\
\text { one of the following clinical } \\
\text { manifestations } \\
\text { - stroke with aphasia and/or paresia } \\
\text { and/or apraxia and/or ataxia } \\
\text { - acute myocardial infarction } \\
\text { - acute renal failure, multi-organ } \\
\text { failure } \\
\text { - coma, seizure } \\
\text { - in case of pregnancy abortion } \\
\text { or stillbirth }\end{array}$ \\
\hline $4=$ lethal & iTTP episode with fatal outcome \\
\hline
\end{tabular}

Abbreviations: iTTP, autoimmune thrombotic thrombocytopenic purpura; LDH, lactate dehydrogenase.

episodes in one individual patient. The significance level was chosen to be 0.05 . We present the rate ratio with its $95 \%$ confidence interval $(\mathrm{CI})$ and its $p$-value. All statistical analyses were performed using SPSS version 22.0 (IBM GmbH, Ehningen, Germany) or the statistical program $\mathrm{R}$ version 3.4.1.

\section{Results}

\section{Recruitment and Characteristics of 70 iTTP Patients}

Since January 2003 until November 2014, a total of 88 patients were seen at the UMC Mainz for suspected iTTP. One young woman died during transfer to the UMC in her first acute iTTP episode (ascertained by an autopsy). Seventy of them demonstrated an ADAMTS13 deficiency and an ADAMTS13 inhibiting autoantibody (-Fig. 1). Sixty-five of the 70 TTP patients had a documented severe ADAMTS13 deficiency $(<10 \%)$ during their acute TTP episode and a detectable ADAMTS13 inhibitor. The other five iTTP patients consulted the UMC Mainz after their first acute episode and suffered from further relapses 


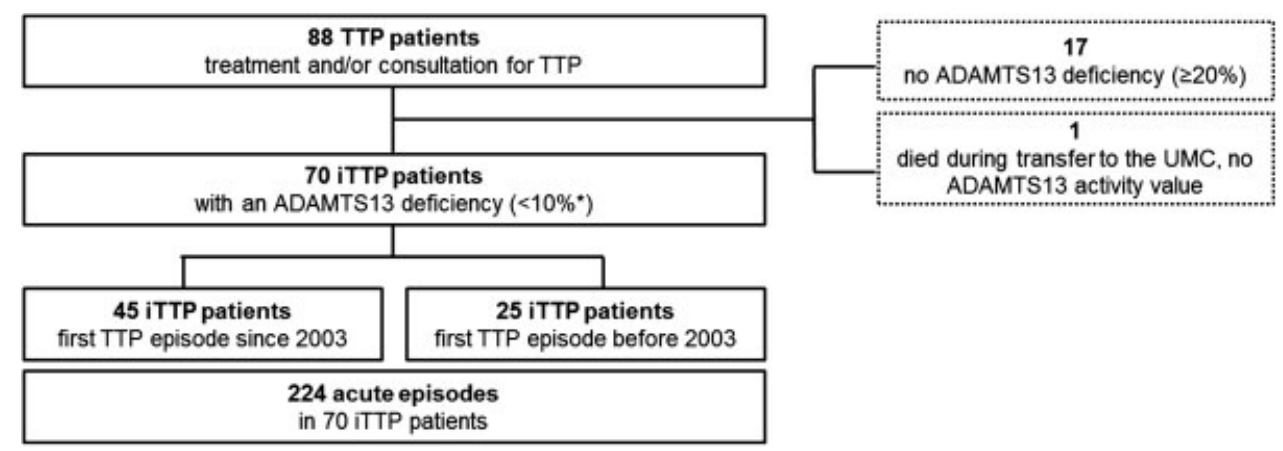

Fig. 1 Patients' recruitment. *Five autoimmune thrombotic thrombocytopenic purpura (iTTP) patients consulted the UMC after having survived their first acute episode. At the time of consultation they showed a disintegrin and metalloprotease with thrombospondin type 1 repeats, member 13 (ADAMTS13) activities of 16, 19, 15, 17 and 18\%, with/ without weak ADAMTS13 inhibitors. All other iTTP patients had documented severe ADAMTS13 deficiency $(<10 \%)$ during acute episode.

until 2014. When consulting in remission, they showed ADAMTS13 activities of $16,19,15,17$ and $18 \%$ with or without weak ADAMTS13 inhibitor titers. The initially treating physicians of those five patients did not have the technical possibilities to measure ADAMTS13 activity or an ADAMTS13 inhibitor on site. Forty-five patients had their first acute iTTP episode during the recruiting period, and 25 iTTP patients had been diagnosed before January 2003. We started our retrospective analysis in 2003, because at that time rituximab was administered for the first time at our institution.

The characteristics of the 70 iTTP patients are shown in - Table 2. The iTTP cohort consisted predominantly of females (77\%) and all patients were Caucasian. Overall, 70

Table 2 Patients' characteristics

\begin{tabular}{|l|l|l|}
\hline Characteristics & No. & $\%$ \\
\hline Total number of iTTP patients & 70 & \\
\hline Gender & & \\
\hline Female & 54 & 77.1 \\
\hline Male & 16 & 22.9 \\
\hline Ethnicity (white Caucasian) & 70 & 100 \\
\hline Total number of acute iTTP episodes & 224 & \\
\hline $\begin{array}{l}\text { Age at time of diagnosis of } \\
\text { first acute iTTP episode, y }\end{array}$ & & \\
\hline Median & 33 & \\
\hline Range & $12-64$ & \\
\hline $\begin{array}{l}\text { Frequency of all acute } \\
\text { episodes per patient }\end{array}$ & & \\
\hline Median, total & 2 & \\
\hline Range & $1-21$ & \\
\hline Median, female & 2.0 & \\
\hline Median, male & 2.5 & \\
\hline Observation time, y & & \\
\hline Median & 8.3 & \\
\hline Range & $0.4-31.9$ & \\
\hline
\end{tabular}

Abbreviation: iTTP, autoimmune thrombotic thrombocytopenic purpura.
iTTP patients suffered from 224 acute episodes over an observation time of 8.3 years (median; range, $0.4-31.9$ years, $\mathrm{IQR}, 4.3-14.3$ years). The median age at diagnosis is 33 years, ranging from 12 to 64 years (IQR, 26-49 years) (- Table 2).

\section{Severity of Acute Bouts and Therapy}

Detailed data for therapy was obtainable in 219 ( - Fig. 2) and for severity in 213 (-Supplementary Table S1, available in the online version) of 224 acute episodes in 70 iTTP patients. No severity and therapy data were accessible in 3 iTTP patients in 4 acute bouts. Thus, detailed data for clinical severity as well as therapy in the respective acute bout were available for 211 of 224 acute episodes in 70 iTTP patients.

Most common were mild symptoms or signs such as fatigue and drowsiness (43.5\%), headaches (26.8\%), petechiae (27.3\%), haematoma (23.4\%), vertigo (12.9\%), nausea (7.2\%) and abdominal pain (6.7\%). Among the more severe symptoms, impairment of consciousness (15.8\%), dyspnoea $(12.9 \%)$, acute renal failure (9.6\%) and haematuria (11.5\%) were most frequent. When counting neurological abnormalities together, they appeared in $34.4 \%$ of all bouts.

During the first iTTP manifestation, the proportion of severe episodes (50\%) was higher than during 1 st relapse (19\%) and all subsequent relapses (13\%) (-Fig. 3). Similarly, moderately severe bouts were more common during 1 st acute episodes (32\%) as compared with 1 st $(22 \%)$ and later $(10 \%)$ relapses. In contrast, mild bouts and mere laboratory abnormalities were more common during 1st relapse (56\%) and further relapses (76\%) than during initial manifestation (17\%) (-Fig. 3 and $\boldsymbol{\sim}$ Supplementary Table S1, available in the online version).

Plasma products were used in 191 of the 219 acute episodes (87\%), prednisolone was administered in 188 (86\%) and rituximab in 69 (32\%) acute bouts. Overall, patients received 12 PEX procedures (median; range, 1-100, IQR, 6-23) for an acute bout. In the first acute episode, $62(91.2 \%)$ iTTP patients received 21.5 PEX procedures (median; range, 2-100, IQR, 12-30). An acute iTTP bout lasted for 29 days (median; range, 1-160 days, IQR, $14-30$ days).

Sixty-nine of of 219 acute bouts in 37 iTTP patients were additionally or exclusively ( 8 acute bouts in 3 different iTTP patients) treated with rituximab, whereas 150 acute bouts in 33 


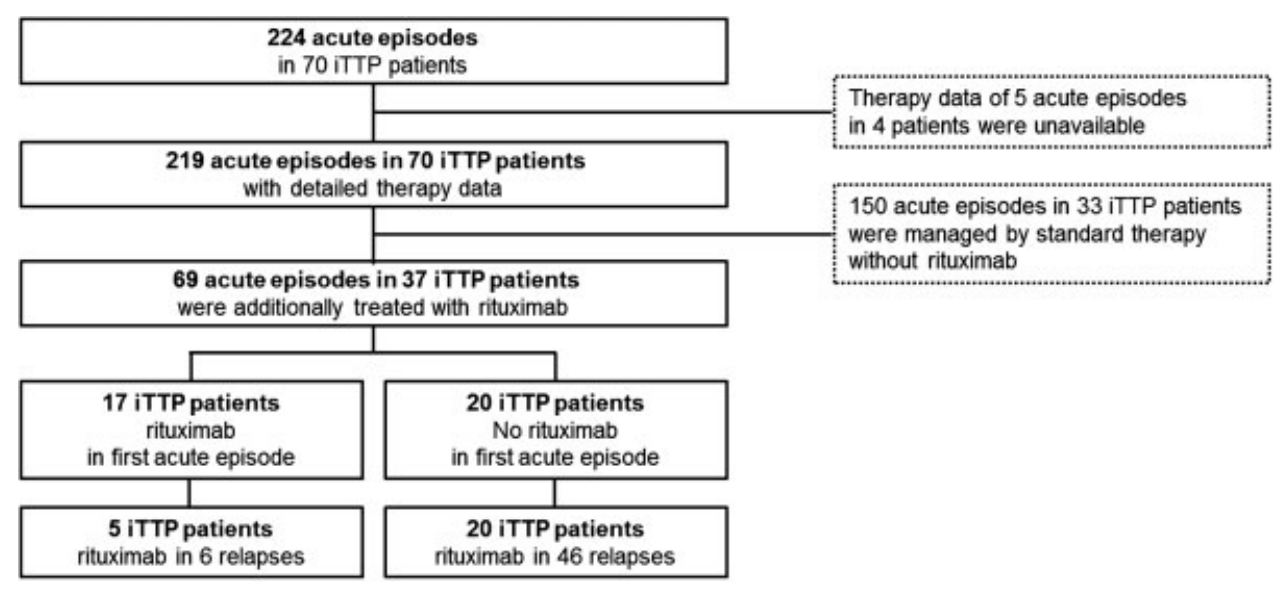

Fig. 2 Treatment of acute autoimmune thrombotic thrombocytopenic purpura (iTTP) episodes.

iTTP patients were not (-Fig. 2). Therefore, $31.5 \%$ of all acute TTP bouts were treated with rituximab in $53 \%$ of iTTP patients.

In this cohort, rituximab has exclusively been used in acute iTTP bouts.

The main indication for rituximab therapy was a refractory episode (58 acute episodes in 37 patients); a second indication was a high tendency to relapse ( 8 acute bouts in 4 patients).

Rituximab therapy was applied in acute iTTP events of all different clinical severity scores. Forty-three per cent of all bouts with severity score of $0,26 \%$ of all mild bouts, $22 \%$ of all moderate bouts, $43 \%$ of all severe bouts and one of the two lethal bouts were treated with additional rituximab (-Supplementary Table S2, available in the online version).

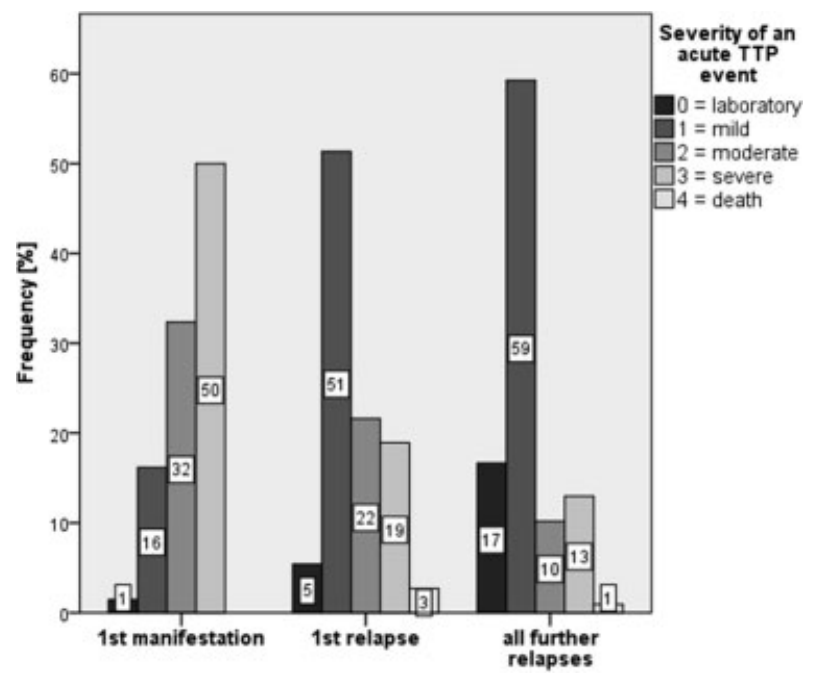

Fig. 3 Frequency of the different clinical severity scores at first, second and all following bouts in 70 autoimmune thrombotic thrombocytopenic purpura (iTTP) patients. During the first iTTP manifestation, the proportion of severe episodes was higher than during 1 st relapse and all subsequent relapses. Absolute numbers in a total of 219 bouts: 1 st manifestation: laboratory (0): $n=1$, mild (1): $n=11$, moderate (2): $n=22$, severe (3): $n=34$; 1 st relapse: laboratory $(0): n=2$, mild (1): $n=19$, moderate $(2): n=8$, severe (3): $n=7$, death (4): $n=1$; all further relapses: laboratory (0): $n=18$, mild (1): $n=64$, moderate (2): $n=11$, severe (3): $n=14$, death (4): $n=1$.

\section{Relapse Rate and Influencing Factors}

Fifty-nine per cent of all 70 iTTP patients had at least one relapse independent of their therapy in acute episodes. Relapse rate ( $\mathbf{- F i g . 4 )}$ is defined as acute recurrent disease episodes in survivors of an initial iTTP bout per 100 patient-months of follow-up. The relapse rate of the whole group was $2.6 \%$ per month. Fifty-four women had a relapse rate of $2.4 \%$ per month and 16 men of 3.5\% per month. Thus, men have a $1.5(95 \% \mathrm{CI}$, $1.11-2.01 ; p=0.009$ ) times higher risk to relapse than women.

We analysed 219 acute episodes concerning possible risk factors for relapses. Smoking status seemed to be associated with higher relapse rate, whereas co-morbidities, including other autoimmune diseases and obesity, did not have an influence on the relapse rate when corrected for gender. With increasing age relapse rate declined.

Relapse rate after acute episodes treated with rituximab was $2.3 \%$ per month in comparison to $2.6 \%$ per month in acute bouts not treated with rituximab ( $\mathbf{- F i g . ~ 4 , ~ A n d e r s o n - G i l l ~ m o d e l , ~}$ $p=0.729$, relative risk, $0.945,95 \% \mathrm{Cl}, 0.687-1.30$ ). Accordingly, rituximab had no significant influence on the relapse rate.

\section{Relapse-Free Survival in $\mathbf{4 5}$ Patients with First iTTP Manifestation Since 2003}

For this analysis, we considered all 45 patients with an initial acute iTTP bout since 2003, the time point when rituximab was first used for this indication at our institution. Seventeen iTTP patients received rituximab during their first acute episode and 20 iTTP patients only during relapses (-Fig. 2). We investigated the relapse-free survival time of these 45 iTTP patients, 17 receiving rituximab compared with 28 not receiving rituximab during their first acute iTTP bout (-Fig. 5). Assessing the relapse-free survival after a first acute episode using Kaplan-Meier analysis, no significant difference between both groups of patients, those treated versus those not treated with rituximab (log-rank test, $p$-value $=0.131$ ) was evident (-Fig. 5). Relapse rate in iTTP treated with rituximab in the initial acute episode was $29 \%$ (5 out of 17) and $50 \%$ ( 14 out of 28 ) in those who did not. The event-free survival in patients treated or not treated with rituximab after 1 year was 94 and 82\%, respectively, and 79 versus 57\%, respectively, after 1,000 days. The median event-free time 


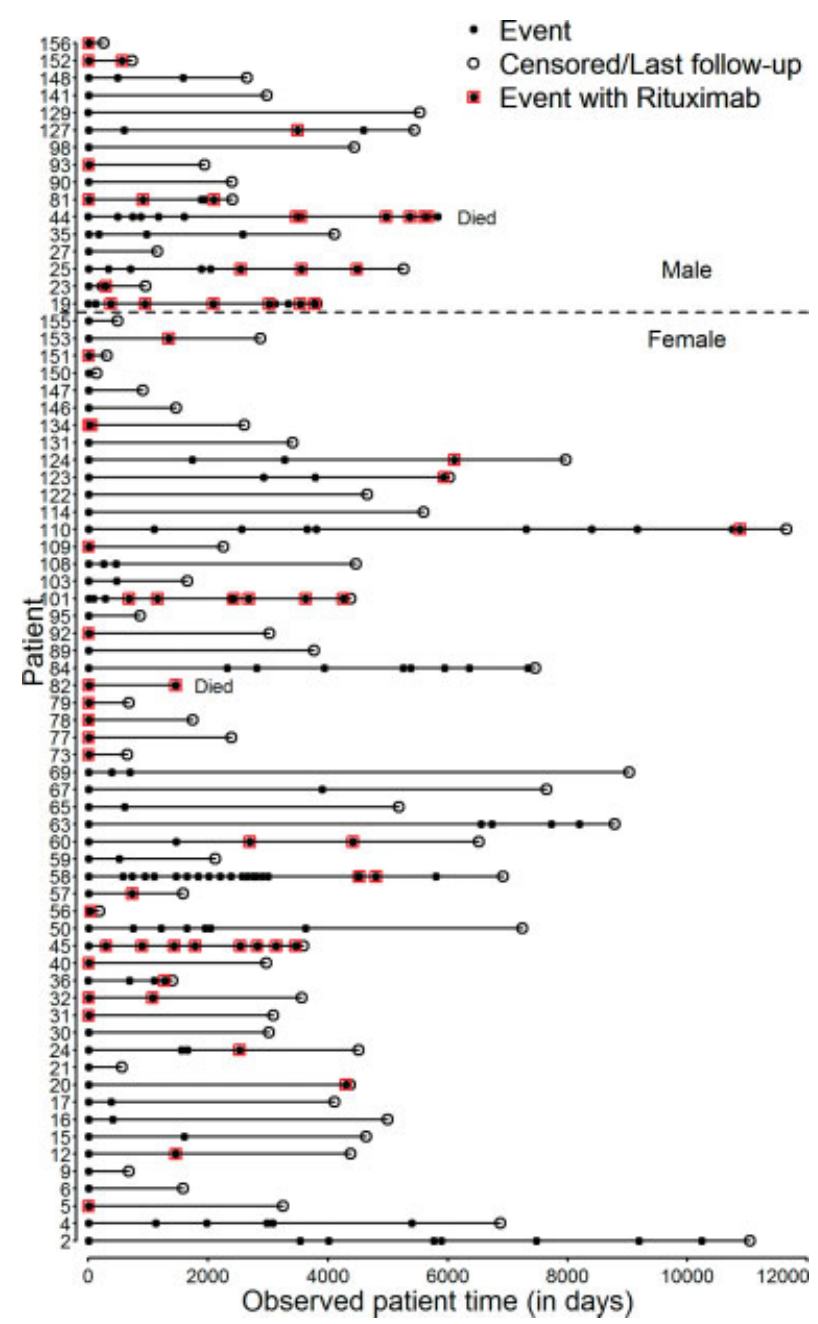

Fig. 4 Event history of all autoimmune thrombotic thrombocytopenic purpura (iTTP) patients with detailed therapy data. Acute iTTP episodes of all 70 patients from the first day of first acute thrombotic thrombocytopenic purpura (TTP) episode until end of observation time in days. Patients were pseudonymized with a number code. Men are listed (above dotted horizontal line no. 19-156) in the upper part and women in the lower part of the figure (below dotted line no. 2-155). Acute iTTP episodes are represented by a black dot. If being treated by rituximab, this circle is bordered by red rectangles. Empty circles represent the last day of observation. Two patients died during a relapse (Died): including one woman following her first acute TTP relapse (no. 82). She had denied plasma products for religious beliefs. One man did not survive his 13th acute TTP episode (no. 44). until first relapse was 1,337 days in patients not treated with rituximab. This value cannot be determined for patients treated with rituximab because less than half of them had a relapse.

\section{Discussion}

Since 2003, rituximab was used in 69 acute episodes as secondline medication in 37 of our 70 patients, generally because they were refractory to standard treatment or showed early relapses. Rituximab has been increasingly used in iTTP over the past 15 years, $7,13,14$ albeit the indications (refractory disease, upfront treatment in all patients, pre-emptive treatment in survivors with recurring severe ADAMTS13 deficiency) are heterogeneous and still debated. ${ }^{15}$ Rituximab was initially used for patients with refractory acute TTP and those with early relapses, and was generally considered to be effective. ${ }^{14,15}$ In our retrospective cohort, $59 \%$ of all 70 iTTP patients had a relapse, independent of whether rituximab was added or not. This is higher than the $40 \%$ reported by Coppo and Froissart and Ferrari et al. ${ }^{16,17}$ Relapse rate was $29 \%$ in 17 iTTP patients treated with rituximab during their first acute episode, and $50 \%$ in those who did not. Chemnitz et al observed a relapse rate of $25 \%$ in their 12 rituximab-treated patients over a 50 -month period. ${ }^{18}$ Scully et al found a relapse rate of only $10 \%$ in her upfrontrituximab-treated cohort, which compared favourably with a rate of $57 \%$ in a historical control group not having received rituximab. ${ }^{19}$ Similar data, relapse rates of $43 \%$ without versus $12.5 \%$ with rituximab, were reported by Page et al in a smaller cohort but with simultaneously treated control group. ${ }^{20}$ According to accumulated data from various cohorts, rituximab may decrease the frequency of subsequent relapses; nonetheless, we can detect only a weak tendency and no significant effect in our cohort. The influence of rituximab seems to be greater in the first year after the initial episode. Our data are similar to that reported by Froissart et al finding no significant difference in the relapse rate of their refractory patients treated versus not treated with rituximab. ${ }^{21}$ Advantage of rituximab seems to lie in a faster recovery and possibly less recurrences during the first year. ${ }^{21-25}$ We can confirm that, in our iTTP patients the event-free-survival after 1 year was $94 \%$ (treated with rituximab) versus $82 \%$ (not treated with rituximab).

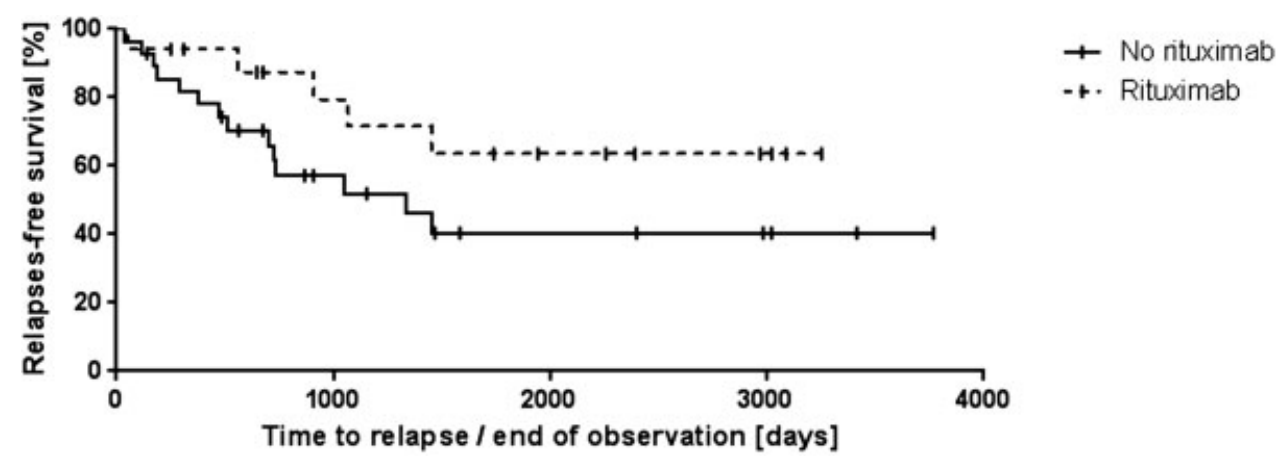

Fig. 5 Kaplan-Meier estimates of relapse-free survival of 45 autoimmune thrombotic thrombocytopenic purpura (iTTP) patients with a first disease bout since 2003. Relapse-free survival of iTTP patients receiving rituximab $(n=17)$ (upper dotted curve) compared with iTTP patients who did not receive rituximab $(n=28)$ (lower curve) during their first acute thrombotic thrombocytopenic purpura (TTP) bout. Vertical bars denote censored patients not having suffered from relapse. There was no statistical difference between these groups $(p$-value $=0.131)$. 
Severe ADAMTS13 deficiency is pathophysiologically strongly linked to the development of clinical disease manifestations. Furthermore, high anti-ADAMTS13 immunoglobulin $\mathrm{G}(\operatorname{IgG})$ antibody titers are associated with increased mortality. ${ }^{26}$ In turn, decrease of anti-ADAMTS13 IgG is directly associated with rituximab therapy, ${ }^{25}$ suppression of B-lymphocytes being detectable for 9 to 15 months. Hence, relapse rate must be carefully watched after this time period. ${ }^{23}$

As it is necessary to achieve remission and reduce relapse frequency, it is desirable to prevent life-threatening relapses at all. In our patients, the initial iTTP bouts were more severe than the later occurring relapses, which have been reported by earlier investigators as well. ${ }^{27}$ This may not be due to the disease itself but rather to increased awareness following the initial diagnosis. Our patients were regularly seen and amenable to early treatment. In a few patients with frequent relapses, rituximab was given after detection of defined laboratory abnormalities even before clinical symptoms arose which led to reduced need of PEX. Early administration of rituximab leads to faster remission and lower number of necessary PEX sessions. ${ }^{22}$ This finally raises the question whether rituximab should be given to any patient with persisting or reappearing severe ADAMTS13 deficiency even before any fall in platelet count or LDH increase is apparent. Hie et al showed that pre-emptive rituximab treatment in asymptomatic survivors of at least one iTTP bout seemed to reduce relapses as compared with patients not receiving rituximab ${ }^{28}$ however, this pre-emptive treatment has been called into question by others. ${ }^{15}$

Moreover, gender plays an important role regarding relapse rate and severity of acute bouts. Generally, men are affected by severe autoimmune diseases more rarely than women. ${ }^{29,30}$ According to our results, men suffered significantly more and also more serious relapses than women. This is similar to other autoimmune diseases such as multiple sclerosis and in systemic lupus erythematous with men suffering from more severe disease course as compared with women. ${ }^{30,31} \mathrm{~A}$ significantly higher tendency to relapse for men has also been described by Fakhouri et al. ${ }^{23}$ Further investigation needs to evaluate whether gender-adapted treatment is necessary.

\section{Strengths and Limitations of this Study}

Our study has several limitations. One is the retrospective data acquisition. In hindsight, missing data or further desirable information could not be collected. On the other side, the long observation time was an advantage over cohorts with shorter follow-up periods. This enabled us to generate relevant results regarding relapse rates. Another limitation is a treatment bias regarding the use of rituximab. Rituximab was predominantly used in iTTP bouts, resistant to conventional PEX and corticosteroid treatment or in patients with a high tendency to relapse. Strength of our study is that iTTP patients given rituximab were compared with simultaneously recruited control iTTP patients, although the two cohorts are small for statistical analysis and not matched by age or sex. A randomized, prospective controlled trial would still be desirable to clarify the role of rituximab but it seems rather unlikely that such a trial will be performed shortly.

In conclusion, we did not detect a significant advantage of rituximab regarding relapse rate, neither during the time between initial acute episode and the first relapse, nor in long-term observation including all acute episodes. Nevertheless, we confirm that rituximab can help to achieve remission in refractory iTTP. Interestingly, we found that men suffer significantly more frequent and considerably more serious acute relapses. Furthermore, initial episodes are characterized by more severe clinical signs compared with the less severe relapses.

\section{What is known about this topic?}

- Rituximab has been increasingly used in iTTP over the past 15 years.

- The first and commonly accepted indication concerns patients refractory to standard treatment with PEX and corticosteroids. Recently, upfront rituximab in any patient with acute iTTP has been proposed based on data suggesting faster remission and shorter hospital stay. Lastly, prophylactic rituximab has been given to survivors of an acute iTTP with recurrent or persistent severe ADAMTS13 deficiency to avoid relapses. No formal indication has been approved.

\section{What does this paper add?}

- First large German cohort of 70 iTTP patients examined for factors influencing the relapse rate.

- Rituximab has no significant effect on the long-term relapse rate in our cohort.

- We report that men suffer significantly more frequent and considerably more serious acute episodes than women.

- Initial acute episodes are characterized by more severe clinical signs compared with the less severe relapses.

\section{Authors' Contributions}

T. Falter: Study concept and design, data analysis, writing of the manuscript and approval. S. Herold: Data acquisition and analysis, writing of the manuscript and approval. V. Weyer: Statistical advice, revision of the manuscript and approval. C. Scheiner: Statistical advice, revision of the manuscript and approval. V. Schmitt: Data acquisition, revision of the manuscript and approval. X. Messmer: Data acquisition and approval. C. von Auer: Revision of the manuscript and approval. P. Wild: Revision of the manuscript and approval. K. Lackner: Revision of the manuscript and approval. B. Lämmle: Data compilation, writing of the manuscript, revision of the manuscript and approval. I. Scharrer: Study initiation, revision of the manuscript, writing of the manuscript and approval. 


\section{Funding}

This study (BMBF 01E01503) as well as Tanja Falter (BMBF 01E01003) were supported by the Federal Ministry of Education and Research.

\section{Conflict of Interest}

The authors declare that they have no conflicts of interest relevant to the manuscript. I. Scharrer is a member of the Data Safety Monitoring Board in the BAX 930 study (investigating recombinant ADAMTS13 infusion in hereditary TTP). She received travel and accommodation support for participating at scientific congresses or meetings from Bayer and NovoNordisk. B. Lämmle is chairman of the Data Safety Monitoring Board in the BAX 930 study (investigating recombinant ADAMTS13 infusion in hereditary TTP). He is on the Advisory Board of Ablynx for the development of caplacizumab. He holds a patent on ADAMTS13 and received travel and accommodation support for participating at scientific congresses or meetings from Baxalta, Siemens, Alexion, Ablynx and Bayer and speaker's fees from Siemens, Bayer and Alexion.

\section{References}

1 Furlan M, Robles R, Lämmle B. Partial purification and characterization of a protease from human plasma cleaving von Willebrand factor to fragments produced by in vivo proteolysis. Blood 1996; 87(10):4223-4234

2 Coppo P, Veyradier A. Thrombotic microangiopathies: towards a pathophysiology-based classification. Cardiovasc Hematol Disord Drug Targets 2009;9(01):36-50

3 Scully M, Hunt BJ, Benjamin S, et al; British Committee for Standards in Haematology. Guidelines on the diagnosis and management of thrombotic thrombocytopenic purpura and other thrombotic microangiopathies. Br J Haematol 2012;158(03): 323-335

4 Kremer Hovinga JA, Vesely SK, Terrell DR, Lämmle B, George JN. Survival and relapse in patients with thrombotic thrombocytopenic purpura. Blood 2010;115(08):1500-1511

5 George JN. How I treat patients with thrombotic thrombocytopenic purpura: 2010. Blood 2010;116(20):4060-4069

6 Rock GA, Shumak KH, Buskard NA, et al; Canadian Apheresis Study Group. Comparison of plasma exchange with plasma infusion in the treatment of thrombotic thrombocytopenic purpura. N Engl J Med 1991;325(06):393-397

7 Scully M. Rituximab in thrombotic thrombocytopenic purpura: medical and financial benefits. Acta Haematol 2015;134(03): 168-169

8 Elliott MA, Heit JA, Pruthi RK, Gastineau DA, Winters JL, Hook CC. Rituximab for refractory and or relapsing thrombotic thrombocytopenic purpura related to immune-mediated severe ADAMTS13-deficiency: a report of four cases and a systematic review of the literature. Eur J Haematol 2009;83 (04):365-372

9 Scully M, Cataland S, Coppo P, et al; International Working Group for Thrombotic Thrombocytopenic Purpura. Consensus on the standardization of terminology in thrombotic thrombocytopenic purpura and related thrombotic microangiopathies. J Thromb Haemost 2017;15(02):312-322

10 Böhm M, Vigh T, Scharrer I. Evaluation and clinical application of a new method for measuring activity of von Willebrand factorcleaving metalloprotease (ADAMTS13). Ann Hematol 2002;81 (08):430-435
11 Kokame K, Nobe Y, Kokubo Y, Okayama A, Miyata T. FRETSVWF73, a first fluorogenic substrate for ADAMTS13 assay. Br J Haematol 2005;129(01):93-100

12 Kremer Hovinga JA, Mottini M, Lämmle B. Measurement of ADAMTS-13 activity in plasma by the FRETS-VWF73 assay: comparison with other assay methods. J Thromb Haemost 2006;4(05):1146-1148

13 Matsumoto M, Fujimura Y, Wada H, et al; For TTP group of Blood Coagulation Abnormalities Research Team, Research on Rare and Intractable Disease supported by Health, Labour, and Welfare Sciences Research Grants. Diagnostic and treatment guidelines for thrombotic thrombocytopenic purpura (TTP) 2017 in Japan. Int J Hematol 2017;106(01):3-15

14 Froissart A, Veyradier A, Hié M, Benhamou Y, Coppo P; French Reference Center for Thrombotic Microangiopathies. Rituximab in autoimmune thrombotic thrombocytopenic purpura: a success story. Eur J Intern Med 2015;26(09):659-665

15 Lim W, Vesely SK, George JN. The role of rituximab in the management of patients with acquired thrombotic thrombocytopenic purpura. Blood 2015;125(10):1526-1531

16 Coppo P, Froissart A; French Reference Center for Thrombotic Microangiopathies. Treatment of thrombotic thrombocytopenic purpura beyond therapeutic plasma exchange. Hematology (Am Soc Hematol Educ Program) 2015;2015:637-643

17 Ferrari S, Scheiflinger F, Rieger M, et al; French Clinical and Biological Network on Adult Thrombotic Microangiopathies. Prognostic value of anti-ADAMTS 13 antibody features (Ig isotype, titer, and inhibitory effect) in a cohort of 35 adult French patients undergoing a first episode of thrombotic microangiopathy with undetectable ADAMTS 13 activity. Blood 2007;109(07):2815-2822

18 Chemnitz JM, Uener J, Hallek M, Scheid C. Long-term follow-up of idiopathic thrombotic thrombocytopenic purpura treated with rituximab. Ann Hematol 2010;89(10):1029-1033

19 Scully M, McDonald V, Cavenagh J, et al. A phase 2 study of the safety and efficacy of rituximab with plasma exchange in acute acquired thrombotic thrombocytopenic purpura. Blood 2011;118 (07):1746-1753

20 Page EE, Kremer Hovinga JA, Terrell DR, Vesely SK, George JN. Rituximab reduces risk for relapse in patients with thrombotic thrombocytopenic purpura. Blood 2016;127(24):3092-3094

21 Froissart A, Buffet M, Veyradier A, et al; French Thrombotic Microangiopathies Reference Center; Experience of the French Thrombotic Microangiopathies Reference Center. Efficacy and safety of first-line rituximab in severe, acquired thrombotic thrombocytopenic purpura with a suboptimal response to plasma exchange. Crit Care Med 2012;40(01):104-111

22 Westwood JP, Webster H, McGuckin S, McDonald V, Machin SJ, Scully M. Rituximab for thrombotic thrombocytopenic purpura: benefit of early administration during acute episodes and use of prophylaxis to prevent relapse. J Thromb Haemost 2013;11(03): $481-490$

23 Fakhouri F, Vernant JP, Veyradier A, et al. Efficiency of curative and prophylactic treatment with rituximab in ADAMTS13-deficient thrombotic thrombocytopenic purpura: a study of 11 cases. Blood 2005;106(06):1932-1937

24 Clark WF, Rock G, Barth D, et al; members of Canadian Apheresis Group. A phase-II sequential case-series study of all patients presenting to four plasma exchange centres with presumed relapsed/refractory thrombotic thrombocytopenic purpura treated with rituximab. Br J Haematol 2015;170(02):208-217

25 Miyakawa Y, Imada K, Ichinohe T, et al. Efficacy and safety of rituximab in Japanese patients with acquired thrombotic thrombocytopenic purpura refractory to conventional therapy. Int J Hematol 2016;104(02):228-235

26 Alwan F, Vendramin C, Vanhoorelbeke K, et al. Presenting ADAMTS13 antibody and antigen levels predict prognosis in immune-mediated thrombotic thrombocytopenic purpura. Blood 2017;130(04):466-471 
27 Rose M, Eldor A. High incidence of relapses in thrombotic thrombocytopenic purpura. Clinical study of 38 patients. Am J Med 1987;83(03):437-444

28 Hie M, Gay J, Galicier L, et al; French Thrombotic Microangiopathies Reference Centre. Preemptive rituximab infusions after remission efficiently prevent relapses in acquired thrombotic thrombocytopenic purpura. Blood 2014;124(02):204-210

29 Boodhoo KD, Liu S, Zuo X. Impact of sex disparities on the clinical manifestations in patients with systemic lupus erythematosus: a systematic review and meta-analysis. Medicine (Baltimore) 2016;95(29):e4272

30 Ortona E, Pierdominici M, Maselli A, Veroni C, Aloisi F, Shoenfeld Y. Sex-based differences in autoimmune diseases. Ann Ist Super Sanita 2016;52(02):205-212

31 Quintero OL, Amador-Patarroyo MJ, Montoya-Ortiz G, RojasVillarraga A, Anaya J-M. Autoimmune disease and gender: plausible mechanisms for the female predominance of autoimmunity. J Autoimmun 2012;38(2-3):J109-J119 